\title{
A MIXED PROBLEM WITH ONLY INTEGRAL BOUNDARY CONDITIONS FOR A HYPERBOLIC EQUATION
}

\author{
ABDELFATAH BOUZIANI
}

Received 29 April 2002

\begin{abstract}
We investigate an initial boundary value problem for a second-order hyperbolic equation with only integral conditions. We show the existence, uniqueness, and continuous dependence of a strongly generalized solution. The proof is based on an energy inequality established in a nonclassical function space, and on the density of the range of the operator associated to the abstract formulation of the studied problem by introducing special smoothing operators.
\end{abstract}

2000 Mathematics Subject Classification: 35L20, 35B45, 35D05, 35B30.

1. Introduction. In recent years, new attention has been devoted to mixed problems for hyperbolic equations with integral conditions. Such conditions appear in case, where for instance, direct measurement quantities are impossible and their mean values are known. Such situations take place in studying, for example, the dynamics of ground waters $[12,13]$. The first investigation of this type of problems goes back to [3] in 1996, in which the author proved the existence, uniqueness, and continuous dependence of the solution upon the data of certain hyperbolic problems with only integral boundary conditions. The proof used in [3] is based, on the one hand, on the method derived by Ladyženskaya [11] to show the existence of the solution, and, on the other hand, on a priori estimates to prove the uniqueness and continuous dependence; these estimates are established by taking the scalar product in $L^{2}$-space of the considered equations and integrodifferential operators constructed for each problem. Later, mixed problems for hyperbolic equations with integral condition(s) were treated in $[2,4,6,7,13,14]$.

In this note, we prove the existence, uniqueness, and continuous dependence of the solution of a mixed problem with only integral boundary conditions for a second-order hyperbolic equation. To this end, we reformulate the stated problem as a problem of solving an operator equation, then we show that the operator generated by the problem is bijective. To prove the injection, we have established an a priori estimate in $B_{2}^{m}$ space, first introduced by the author (see, e.g., [4, 6]). Thanks to this space, we have used the same operator of multiplication employed to obtain estimates for a secondorder hyperbolic equation with classical boundary conditions, without having recourse to constructing an appropriate integrodifferential operator for the posed problem. This confirms what it is noted in [6] concerning the importance of the use of such space to solve a large class of problems with integral boundary conditions. In order to prove the surjection of the operator, we have constructed particular smoothing operators with 
respect to $t$. These operators can be used to solve a large class of evolution problems possessing second-order derivative in time.

2. Statement of the problem and notations. In the rectangular domain $Q=(a, b) \times$ $(0, T)$, we consider the following problem: find the function $v=v(x, t)$ satisfying

$$
\begin{gathered}
\mathscr{L} v=\frac{\partial^{2} v}{\partial t^{2}}-\frac{\partial}{\partial x}\left(p(x, t) \frac{\partial v}{\partial x}\right)+q(x, t) \frac{\partial v}{\partial t}+r(x, t) \frac{\partial v}{\partial x} \\
+s(x, t) v=\mathrm{f}(x, t), \quad(x, t) \in Q, \\
\ell_{0} v=v(x, 0)=v_{0}(x), \quad x \in(a, b), \\
\ell_{1} v=\frac{\partial v(x, 0)}{\partial t}=v_{1}(x), \quad x \in(a, b), \\
\int_{a}^{b} v(x, t) d x=E(t), \quad t \in(0, T), \\
\int_{a}^{b} x v(x, t) d x=M(t), \quad t \in(0, T),
\end{gathered}
$$

with

$$
\begin{array}{ll}
\int_{a}^{b} v_{0}(x) d x=E(0), & \int_{a}^{b} x v_{0}(x) d x=M(0), \\
\int_{a}^{b} v_{1}(x) d x=E^{\prime}(0), & \int_{a}^{b} x v_{1}(x) d x=M^{\prime}(0),
\end{array}
$$

where $v_{0}, v_{1}, E, M, \mathrm{f}, p, q, r$, and $s$ are known functions and $T>0, a$, and $b$ are given constants.

Assumption 2.1. We will assume, for $(x, t) \in \bar{Q}$, that

$$
\begin{gathered}
0<c_{0} \leq p(x, t) \leq c_{1}, \quad\left|\frac{\partial p}{\partial t}\right| \leq c_{2}, \quad\left|\frac{\partial p}{\partial x}\right| \leq c_{3}, \quad q(x, t) \geq 0, \\
\left|\frac{\partial q}{\partial x}\right| \leq c_{4}, \quad|r(x, t)| \leq c_{5}, \quad\left|\frac{\partial r}{\partial x}\right| \leq c_{6}, \quad|s(x, t)| \leq c_{7} .
\end{gathered}
$$

In Assumption 2.1 and throughout, $c_{i}$ are positive constants.

Since integral boundary conditions are inhomogeneous, it is convenient to convert problem (2.1) to an equivalent problem with homogeneous integral conditions. For this, we introduce a new function $u(x, t)$ representing the deviation of the function $v(x, t)$ from the function

$$
\begin{aligned}
U(x, t)= & 12(x-a)(3 x-a-2 b) \frac{M(t)}{(b-a)^{4}} \\
& -\left(18(x-a)^{2}-12(x-a)(b-a)-(b-a)^{2}\right) \frac{E(t)}{(b-a)^{3}} .
\end{aligned}
$$


Then, our problem becomes as follows: find a function $u=u(x, t)$ satisfying

$$
\begin{gathered}
\mathscr{L} u=\mathrm{f}(x, t)-\mathscr{L} U=f(x, t), \quad(x, t) \in Q, \\
\ell_{0} u=u(x, 0)=u_{0}(x), \quad x \in(a, b), \\
\ell_{1} u=\frac{\partial u(x, 0)}{\partial t}=u_{1}(x), \quad x \in(a, b), \\
\int_{a}^{b} u(x, t) d x=0, \quad t \in(0, T), \\
\int_{a}^{b} x u(x, t) d x=0, \quad t \in(0, T),
\end{gathered}
$$

with

$$
\int_{a}^{b} u_{i}(x) d x=0, \quad \int_{a}^{b} x u_{i}(x) d x=0 \quad(i=0,1) .
$$

We introduce the appropriate function spaces that will be used in the rest of the note. Let $H$ be a Hilbert space with a norm $\|\cdot\|_{H}$.

DEFINITION 2.2. (i) Denote by $L^{2}(0, T ; H)$ the set of all measurable abstract functions $u(\cdot, t)$ from $(0, T)$ into $H$ such that

$$
\|u\|_{L^{2}(0, T ; H)}=\left(\int_{0}^{T}\|u(\cdot, t)\|_{H}^{2} d t\right)^{1 / 2}<\infty .
$$

(ii) Let $C(0, T ; H)$ be the set of all continuous functions $u(\cdot, t):(0, T) \rightarrow H$ with

$$
\|u\|_{C(0, T ; H)}=\max _{0 \leq t \leq T}\|u(\cdot, t)\|_{H}<\infty .
$$

Definition 2.3. Denote by $B_{2}^{m}(a, b)$ the space equipped with the scalar product

$$
(u, w)_{B_{2}^{m}(a, b)}=\int_{a}^{b} \mathfrak{J}_{x}^{m} u \cdot \mathfrak{I}_{x}^{m} v d x
$$

and the associated norm

$$
\|u\|_{B_{2}^{m}(a, b)}=\left(\int_{a}^{b}\left(\mathfrak{J}_{x}^{m} u\right)^{2} d x\right)^{1 / 2},
$$

where

$$
\mathfrak{J}_{x}^{m} u=\frac{1}{(m-1) !} \int_{a}^{x}(x-\xi)^{m-1} u(\xi, \cdot) d \xi, \quad m \geq 1 .
$$

LEMMA 2.4. For $m \geq 1$, the following inequality holds:

$$
\|u\|_{B_{2}^{m}(a, b)}^{2} \leq \frac{(b-a)^{2}}{2}\|u\|_{B_{2}^{m-1}(a, b)}^{2} .
$$

When $m=0, B_{2}^{0}(a, b)=L^{2}(a, b)$. 
DEFINITION 2.5. Let $B_{2}^{n, w}(0, T)$ be the weighted $B_{2}^{n}$-space equipped with the norm

$$
\|u\|_{B_{2}^{n, w}(0, T)}=\left(\int_{0}^{T} w(t)\left(\mathfrak{J}_{t}^{n} u\right)^{2} d t\right)^{1 / 2} .
$$

In this note, $w(t)=e^{-c t}$.

DEFINITION 2.6. Write $B_{2}^{n}(0, T ; H)$ (resp., $B_{2}^{n, w}(0, T ; H)$ ) for the space of functions from $(0, T)$ into $H$, which is a $B_{2}^{n}$-space (resp., a $B_{2}^{n, w}$-space, ) for the measure $d t$. It is a Hilbert space for the norm

$$
\|u\|_{B_{2}^{n}(0, T ; H)}=\left(\int_{0}^{T}\left(\mathfrak{J}_{t}^{n}\|u(\cdot, \tau)\|_{H}\right)^{2} d t\right)^{1 / 2},
$$

respectively,

$$
\|u\|_{B_{2}^{n, w}(0, T ; H)}=\left(\int_{0}^{T} w(t)\left(\mathfrak{J}_{t}^{n}\|u(\cdot, \tau)\|_{H}\right)^{2} d t\right)^{1 / 2} .
$$

Problem (2.5), (2.6), (2.7), (2.8), and (2.9) can be written in the following abstract form:

$$
L u=\left(f, u_{0}, u_{1}\right)
$$

where $L=\left(\mathscr{L}, \ell_{0}, \ell_{1}\right)$. The operator $L$, with domain $D(L)$, acts from $B$ to $F$, where $D(L)$ is the set of all functions $u \in L^{2}\left(0, T ; B_{2}^{1}(a, b)\right)$ for which $\partial^{i} u / \partial t^{i}, \partial^{i} u / \partial x^{i}(i=1,2)$, and $\partial^{3} u / \partial x \partial t^{2}$ belong to $L^{2}\left(0, T ; B_{2}^{1}(a, b)\right)$ and $u$ satisfies conditions (2.8) and (2.9), $B$ is the Banach space obtained by the closure of $D(L)$ in the norm

$$
\|u\|_{B}=\left(\|u\|_{C\left(0, T ; L^{2}(a, b)\right)}^{2}+\left\|\frac{\partial u}{\partial t}\right\|_{C\left(0, T ; B_{2}^{1}(a, b)\right)}^{2}\right)^{1 / 2},
$$

and $F$ is the Hilbert space $L^{2}\left(0, T ; B_{2}^{1}(a, b)\right) \times L^{2}(a, b) \times B_{2}^{1}(a, b)$.

Let $\bar{L}$ be the closure of $L$ with domain $D(\bar{L})$.

DEFINITION 2.7. A solution of the abstract equation

$$
\bar{L} u=\left(f, u_{0}, u_{1}\right)
$$

is called a strongly generalized solution of problem (2.5), (2.6), (2.7), (2.8), and (2.9).

3. Uniqueness and continuous dependence. We first establish an energy inequality; the uniqueness and continuous dependence of the solution with respect to the data are immediate consequences.

THEOREM 3.1. Under Assumption 2.1, the following inequality holds for any function $u \in D(L)$ :

$$
\|u\|_{B} \leq c\|L u\|_{F},
$$

where $c$ is a positive constant independent of $u$. 
Proof. Taking the scalar product, in $B_{2}^{1}(a, b)$, of (2.5) and $\partial u / \partial t$ and integrating by parts, we get

$$
\begin{aligned}
2 \int_{a}^{b} q\left(\mathfrak{J}_{x} \frac{\partial u}{\partial t}\right)^{2} d x+\frac{\partial}{\partial t}\left(\left\|\frac{\partial u(\cdot, t)}{\partial t}\right\|_{B_{2}^{1}(a, b)}^{2}+\int_{a}^{b} p u^{2} d x\right) \\
=2\left(f(\cdot, t), \frac{\partial u(\cdot, t)}{\partial t}\right)_{B_{2}^{1}(a, b)}+\int_{a}^{b} \frac{\partial p}{\partial t} u^{2} d x-2 \int_{a}^{b} \frac{\partial p}{\partial x} u \mathfrak{J}_{x} \frac{\partial u}{\partial t} d x \\
\quad-2 \int_{a}^{b} \frac{\partial q}{\partial x} \mathfrak{I}_{x} \frac{\partial u}{\partial t} \mathfrak{J}_{x}^{2} \frac{\partial u}{\partial t} d x-2 \int_{a}^{b} r u \mathfrak{J}_{x} \frac{\partial u}{\partial t} d x-2 \int_{a}^{b} \frac{\partial r}{\partial x} u \mathfrak{I}_{x}^{2} \frac{\partial u}{\partial t} d x \\
\quad-2 \int_{a}^{b} \mathfrak{I}_{x}(s u) \mathfrak{I}_{x} \frac{\partial u}{\partial t} d x .
\end{aligned}
$$

According to the $\varepsilon$-inequality, the right-hand side of (3.2) is bounded by

$$
\begin{gathered}
\|f(\cdot, t)\|_{B_{2}^{1}(a, b)}^{2}+\int_{a}^{b}\left(\frac{\partial p}{\partial t}+\left(\frac{\partial p}{\partial x}\right)^{2}+r^{2}+\left(\frac{\partial r}{\partial x}\right)^{2}\right) u^{2} d x+4\left\|\frac{\partial u(\cdot, t)}{\partial t}\right\|_{B_{2}^{1}(a, b)}^{2} \\
+2\left\|\frac{\partial u(\cdot, t)}{\partial t}\right\|_{B_{2}^{2}(a, b)}^{2}+\int_{a}^{b}\left(\frac{\partial q}{\partial x}\right)^{2}\left(\mathfrak{J}_{x} \frac{\partial u}{\partial t}\right)^{2} d x+\int_{a}^{b}\left(\mathfrak{J}_{x}(s u)\right)^{2} d x .
\end{gathered}
$$

Substituting (3.3) into (3.2) and integrating the result over $(0, \tau)$, with $0 \leq \tau \leq T$, we obtain, by using inequality (2.16) for $m=1$ and $m=2$,

$$
\begin{aligned}
2 \int_{0}^{\tau} \int_{a}^{b} q\left(\mathfrak{J}_{x} \frac{\partial u}{\partial t}\right)^{2} d x d t+\int_{a}^{b} p(x, \tau) u^{2}(x, \tau) d x+\left\|\frac{\partial u(\cdot, \tau)}{\partial t}\right\|_{B_{2}^{1}(a, b)}^{2} \\
\leq \int_{0}^{\tau}\|f(\cdot, t)\|_{B_{2}^{1}(a, b)}^{2} d t+\int_{a}^{b} p(\cdot, 0) u_{0}^{2} d x+\left\|u_{1}\right\|_{B_{2}^{1}(a, b)}^{2} \\
\quad+\int_{0}^{\tau} \int_{a}^{b}\left(\frac{\partial p}{\partial t}+\left(\frac{\partial p}{\partial x}\right)^{2}+r^{2}+\left(\frac{\partial r}{\partial x}\right)^{2}+\frac{(b-a)^{2}}{2} s^{2}\right) u^{2} d x d t \\
\quad+\left(4+(b-a)^{2}\right) \int_{0}^{\tau}\left\|\frac{\partial u(\cdot, t)}{\partial t}\right\|_{B_{2}^{1}(a, b)}^{2} d t+\int_{0}^{\tau} \int_{a}^{b}\left(\frac{\partial q}{\partial x}\right)^{2}\left(\mathfrak{J}_{x} \frac{\partial u}{\partial t}\right)^{2} d x d t .
\end{aligned}
$$

By virtue of Assumption 2.1, we have

$$
\begin{aligned}
\| u(\cdot, \tau) & \left\|_{L^{2}(a, b)}^{2}+\right\| \frac{\partial u(\cdot, \tau)}{\partial t} \|_{B_{2}^{1}(a, b)}^{2} \\
\leq & c_{8}\left(\int_{0}^{\tau}\|f(\cdot, t)\|_{B_{2}^{1}(a, b)}^{2} d t+\left\|u_{0}\right\|_{L^{2}(a, b)}^{2}+\left\|u_{1}\right\|_{B_{2}^{1}(a, b)}^{2}\right) \\
& +c_{9} \int_{0}^{\tau}\left(\|u(\cdot, t)\|_{L^{2}(a, b)}^{2}+\left\|\frac{\partial u(\cdot, t)}{\partial t}\right\|_{B_{2}^{1}(a, b)}^{2}\right) d t,
\end{aligned}
$$


where

$$
\begin{gathered}
c_{8}=\frac{\max \left(1, c_{1}\right)}{\min \left(1, c_{0}\right)}, \\
c_{9}=\frac{\max \left(c_{2}+c_{3}^{2}+c_{5}^{2}+c_{6}^{2}+\left((b-a)^{2} / 2\right) c_{7}^{2}, 4+(b-a)^{2}+c_{4}^{2}\right)}{\min \left(1, c_{0}\right)} .
\end{gathered}
$$

The application of Gronwall's lemma implies

$$
\begin{aligned}
& \|u(\cdot, \tau)\|_{L^{2}(a, b)}^{2}+\left\|\frac{\partial u(\cdot, \tau)}{\partial t}\right\|_{B_{2}^{1}(a, b)}^{2} \\
& \quad \leq c_{8} \exp \left(c_{9} T\right)\left(\|f\|_{L^{2}\left(0, T ; B_{2}^{1}(a, b)\right)}^{2}+\left\|u_{0}\right\|_{L^{2}(a, b)}^{2}+\left\|u_{1}\right\|_{B_{2}^{1}(a, b)}^{2}\right) .
\end{aligned}
$$

The right-hand side here is independent of $\tau$; thus taking the upper bound for $0 \leq \tau \leq T$ in the left-hand side, we obtain estimate (3.1), where $c=c_{8}^{1 / 2} \exp \left(c_{8} T / 2\right)$.

As we have no information concerning $R(L)$ expect that $R(L) \subset F$, we must extend $L$, so that estimate (3.1) holds for the extension and its range is the whole space. We first state the following result.

Proposition 3.2. Under the hypotheses of Theorem 3.1, the operator L acting from $B$ into $F$ has a closure.

Proof. The proof is analogous to that of [5, Proposition 1].

The next corollary follows from Theorem 3.1 and Proposition 3.2.

COROLLARY 3.3. Under the assumptions of Theorem 3.1, the operator $\bar{L}$ has on $R(\bar{L})$ a continuous inverse $\bar{L}^{-1}$, that is, there exists a constant $c>0$ such that

$$
\|u\|_{B} \leq c\|\bar{L} u\|_{F}, \quad u \in D(\bar{L}) .
$$

Proof. Inequality (3.8) can be obtained by passing to the limit in (3.1).

COROLLARY 3.4. If problem (2.5), (2.6), (2.7), (2.8), and (2.9) has a strongly generalized solution, then this solution is unique and depends continuously on $\left(f, u_{0}, u_{1}\right)$.

COROLLARY 3.5. The range of the operator $\bar{L}$ equals the closure of the range of the operator $L$, that is, $R(\bar{L})=\overline{R(L)}$.

Proof. The proof is the same as that of [5, Corollary 2.5].

4. Existence of the solution. Now, we are able to state and prove our main result.

TheOREM 4.1. Assume that the hypothesis of Theorem 3.1 holds. Moreover, it is assumed that the coefficients $\partial^{2} p / \partial t^{2}, \partial^{3} p / \partial t^{2} \partial x$ are bounded. Then, for $f \in L^{2}(0, T$; $\left.B_{2}^{1}(a, b)\right), u_{0} \in L^{2}(a, b)$, and $u_{1} \in B_{2}^{1}(a, b)$, there exists a unique strongly generalized solution $u=\bar{L}^{-1}\left(f, u_{0}, u_{1}\right)=\overline{L^{-1}}\left(f, u_{0}, u_{1}\right)$ of problem (2.5), (2.6), (2.7), (2.8), and (2.9) 
that satisfies

$$
\|u\|_{B} \leq c\left(\|f\|_{L^{2}\left(0, T ; B_{2}^{1}(a, b)\right)}+\left\|u_{0}\right\|_{L^{2}(a, b)}+\left\|u_{1}\right\|_{B_{2}^{1}(a, b)}\right),
$$

where $c$ is a positive constant independent of $u$.

Proof. By virtue of Corollary 3.5, we conclude that it is sufficient to prove that $\overline{R(L)}=F$. To this end, we will first establish the density for a particular case in which $L$ is reduced to $L_{0}$ and $u \in D_{0}\left(L_{0}\right)$, where $L_{0}=\left(\mathscr{L}_{0}, \ell_{1}, \ell_{2}\right), \mathscr{L}_{0}$ is the principal part of $\mathscr{L}$, that is, $\mathscr{L}_{0}=\partial^{2} / \partial t^{2}-(\partial / \partial x)(p(x, t)(\partial / \partial x)), D_{0}\left(L_{0}\right)=D_{0}(L)$, and $D_{0}(L)$ is the set of all functions $u \in D(L)$ for which $\ell_{i} u=0(i=0,1)$.

Proposition 4.2. Under the hypotheses of Theorem 4.1, if

$$
\left(\mathscr{L}_{0} u, \omega\right)_{L^{2}\left(0, T ; B_{2}^{1}(a, b)\right)}=0
$$

for arbitrary $u \in D_{0}\left(L_{0}\right)$ and some $\omega \in L^{2}\left(0, T ; B_{2}^{1}(a, b)\right)$, then $\omega$ vanishes almost everywhere in $Q$.

Suppose for a moment that Proposition 4.2 has been established and return to the proof of Theorem 4.1. Let the element $\left(f, u_{0}, u_{1}\right)$ of $F=L^{2}\left(0, T ; B_{2}^{1}(a, b)\right) \times L^{2}(a, b) \times$ $B_{2}^{1}(a, b)$ be orthogonal to $R\left(L_{0}\right)$, that is,

$$
\left(\mathscr{L}_{0} u, f\right)_{L^{2}\left(0, T ; B_{2}^{1}(a, b)\right)}+\left(\ell_{0} u, u_{0}\right)_{L^{2}(a, b)}+\left(\ell_{1} u, u_{1}\right)_{B_{2}^{1}(a, b)}=0, \quad u \in D\left(L_{0}\right) .
$$

If $u \in D_{0}\left(L_{0}\right)$, it follows from Proposition 4.2 that $f$ vanishes almost everywhere in $Q$. Hence

$$
\left(\ell_{0} u, u_{0}\right)_{L^{2}(a, b)}+\left(\ell_{1} u, u_{1}\right)_{B_{2}^{1}(a, b)}=0, \quad u \in D\left(L_{0}\right)
$$

But $R\left(\ell_{1}\right)$ and $R\left(\ell_{2}\right)$ are everywhere dense in $L^{2}(a, b)$ and $B_{2}^{1}(a, b)$, respectively. So, $\omega_{1}=\omega_{2}=0$, from which we conclude that $\overline{R\left(L_{0}\right)}=F$.

We turn back to the general case. The operator $L-L_{0}$ maps continuously $B$ into $F$; then Theorem 4.1 can be proved by the method of continuation with respect to the parameter. We will not describe it here; however we refer the reader, for instance, to [8].

To complete the proof of Theorem 4.1, it remains to establish the proof of Proposition 4.2 .

Proof of Proposition 4.2. We need to introduce the family of smoothing operators with respect to $t$ :

$$
\begin{aligned}
& \varrho_{\varepsilon}^{-2} \theta=\frac{1}{\sqrt{\varepsilon}} \int_{0}^{t} \sin \frac{1}{\sqrt{\varepsilon}}(t-\tau) \theta(x, \tau) d \tau, \quad \varepsilon>0, \\
& \left(\varrho_{\varepsilon}^{-2}\right)^{*} \theta=\frac{1}{\sqrt{\varepsilon}} \int_{t}^{T} \sin \frac{1}{\sqrt{\varepsilon}}(\tau-t) \theta(x, \tau) d \tau, \quad \varepsilon>0 .
\end{aligned}
$$


These operators provide the solutions of the problems

$$
\begin{gathered}
\varepsilon \frac{\partial^{2} \varrho_{\varepsilon}^{-2} \theta(x, t)}{\partial t^{2}}+\varrho_{\varepsilon}^{-2} \theta(x, t)=\theta(x, t), \\
\varrho_{\varepsilon}^{-2} \theta(x, 0)=0, \quad \frac{\partial \varrho_{\varepsilon}^{-2} \theta(x, 0)}{\partial t}=0, \\
\varepsilon \frac{\partial^{2}\left(\varrho_{\varepsilon}^{-2}\right)^{*} \theta(x, t)}{\partial t^{2}}+\left(\varrho_{\varepsilon}^{-2}\right)^{*}(x, t)=\theta(x, t), \\
\left(\varrho_{\varepsilon}^{-2}\right)^{*} \theta(x, T)=0, \quad \frac{\partial\left(\varrho_{\varepsilon}^{-2}\right)^{*} \theta(x, T)}{\partial t}=0,
\end{gathered}
$$

respectively. They have the following properties.

LEMMA 4.3. If $\theta \in L^{2}(0, T)$, then

(1) $\varrho_{\varepsilon}^{-2} \theta \in H^{2}(0, T)$ and $\varrho_{\varepsilon}^{-2} \theta(x, 0)=0, \partial \varrho_{\varepsilon}^{-2} \theta(x, 0) / \partial t=0$,

(2) $\left(\varrho_{\varepsilon}^{-2}\right)^{*} \theta \in H^{2}(0, T)$ and $\left(\varrho_{\varepsilon}^{-2}\right)^{*} \theta(x, T)=0, \partial\left(\varrho_{\varepsilon}^{-2}\right) * \theta(x, T) / \partial t=0$.

LEMMA 4.4. If $\theta$ and $g$ are in $L^{2}(0, T)$, then

$$
\int_{0}^{T} \varrho_{\varepsilon}^{-2} \theta \cdot g d t=\int_{0}^{T} \theta \cdot\left(\varrho_{\varepsilon}^{-2}\right)^{*} g d t
$$

Lemmas 4.3 and 4.4 are proved directly by using the definitions of the operators $\varrho_{\varepsilon}^{-2}$ and $\left(\varrho_{\varepsilon}^{-2}\right)^{*}$.

LEMMA 4.5. For all $\theta \in L^{2}(0, T)$ such that $\partial^{2} \theta / \partial t^{2}$ is in $L^{2}(0, T)$, the following identity holds:

$$
\varrho_{\varepsilon}^{-2} \frac{\partial^{2} \theta}{\partial \tau^{2}}=\frac{\partial^{2}\left(\varrho_{\varepsilon}^{-2}\right) \theta}{\partial t^{2}}-\frac{1}{\varepsilon} \theta(x, 0) \cos \frac{t}{\sqrt{\varepsilon}}-\frac{1}{\sqrt{\varepsilon}} \frac{\partial \theta(x, 0)}{\partial t} \int_{0}^{t} \sin \frac{s}{\sqrt{\varepsilon}} d s .
$$

To prove this lemma, it suffices to integrate by parts the expression $\varrho_{\varepsilon}^{-2}\left(\partial^{2} \theta / \partial \tau^{2}\right)$.

LEMMA 4.6. For all $\theta \in L^{2}(0, T ; H)$,

(1) $\int_{0}^{T}\left\|\varrho_{\varepsilon}^{-2} \theta\right\|^{2} d t \leq \int_{0}^{T}\|\theta\|_{H}^{2} d t$ and $\int_{0}^{T}\left\|\varrho_{\varepsilon}^{-2} \theta-\theta\right\|_{H}^{2} d t \rightarrow 0$, when $\varepsilon \rightarrow 0$,

(2) $\int_{0}^{T}\left\|\left(\varrho_{\varepsilon}^{-2}\right)^{*}\right\|_{H}^{2} d t \leq \int_{0}^{T}\|\theta\|_{H}^{2} d t$ and $\int_{0}^{T}\left\|\left(\varrho_{\varepsilon}^{-2}\right)^{*}-\theta\right\|_{H}^{2} d t \rightarrow 0$, when $\varepsilon \rightarrow 0$.

The proof of Lemma 4.6 is similar to that of [1, Lemma 2.18].

LEMMA 4.7. Set $P(t)=(\partial / \partial x)(p(x, t)(\partial / \partial x))$; then the following relation holds:

$$
P(t) \varrho_{\varepsilon}^{-2}=\varrho_{\varepsilon}^{-2} P(\tau) \varrho_{\varepsilon}^{-2}+\varrho_{\varepsilon}^{-2} P^{\prime \prime}(\tau) \varrho_{\varepsilon}^{-2},
$$

where $P^{\prime \prime}(\tau)=(\partial / \partial x)\left(\left(\partial^{2} p(x, t) / \partial t^{2}\right)(\partial / \partial x)\right)$.

Lemma 4.5 is proved directly by integrating by parts $\varrho_{\varepsilon}^{-2} P(\tau)$.

Note that similar operators related to equations of order one in time and to operational equations are established in $[9,10]$.

From (4.2), we have

$$
\left(\frac{\partial^{2} u}{\partial t^{2}}, \omega\right)_{L^{2}\left(0, T ; B_{2}^{1}(a, b)\right)}=(P u, \omega)_{L^{2}\left(0, T ; B_{2}^{1}(a, b)\right)} .
$$


We replace $u$ by $\varrho_{\varepsilon}^{-2} u$ in (4.11) and apply Lemma 4.5 to the left-hand side and Lemma 4.7 to the right-hand side; we get

$$
\left(\varrho_{\varepsilon}^{-2} \frac{\partial^{2} u}{\partial \tau^{2}}, \omega\right)_{L^{2}\left(0, T ; B_{2}^{1}(a, b)\right)}=\left(\varrho_{\varepsilon}^{-2} P \varrho_{\varepsilon}^{-2} u+\varepsilon \varrho_{\varepsilon}^{-2} P^{\prime \prime} \varrho_{\varepsilon}^{-2} u, \omega\right)_{L^{2}\left(0, T ; B_{2}^{1}(a, b)\right)}
$$

According to Lemma 4.4, we obtain

$$
\left(\frac{\partial^{2} u}{\partial t^{2}},\left(\varrho_{\varepsilon}^{-2}\right)^{*} \omega\right)_{L^{2}\left(0, T ; B_{2}^{1}(a, b)\right)}=\left(P \varrho_{\varepsilon}^{-2} u+\varepsilon P^{\prime \prime} \varrho_{\varepsilon}^{-2} u,\left(\varrho_{\varepsilon}^{-2}\right)^{*} \omega\right)_{L^{2}\left(0, T ; B_{2}^{1}(a, b)\right)},
$$

from which we have

$$
\begin{aligned}
\int_{Q} u & \frac{\partial^{2}\left(\varrho_{\varepsilon}^{-2}\right)^{*} \mathfrak{J}_{x}^{*}\left(\mathfrak{J}_{\xi} \omega\right)}{\partial t^{2}} d x d t \\
& =\int_{Q}\left(P(t) \varrho_{\varepsilon}^{-2} u+\varepsilon P^{\prime \prime}(t) \varrho_{\varepsilon}^{-2} u\right)\left(\varrho_{\varepsilon}^{-2}\right)^{*} \mathfrak{J}_{x}^{*}\left(\mathfrak{J}_{\xi} \omega\right) d x d t
\end{aligned}
$$

The operator $P(t)$ with conditions (2.8) and (2.9) has, on $L^{2}(0, T)$, a continuous inverse defined by

$$
\begin{aligned}
P^{-1}(t) g= & \int_{a}^{x} \frac{d \xi}{p(\xi, t)} \int_{a}^{\xi} g(\zeta, t) d \zeta+\frac{1}{(b-a) \int_{a}^{b}((a-x)(b-x) / p(x, t)) d x} \\
& \times\left\{\left(\int_{a}^{b} \frac{\left(b^{2}-x^{2}\right)}{p(x, t)} d x\right)\left(\int_{a}^{b} \frac{(b-x)}{p(x, t)} d x \int_{a}^{x} g(\xi, t) d \xi\right)\right. \\
& -\left(\int_{a}^{b} \frac{(b-x)}{p(x, t)} d x\right)\left(\int_{a}^{b} \frac{\left(b^{2}-x^{2}\right)}{p(x, t)} d x \int_{a}^{x} g(\xi, t) d \xi\right) \\
& \left.-(b-a)\left(\int_{a}^{b} \frac{(a-x)(b-x)}{p(x, t)} d x \int_{a}^{x} g(\xi, t) d \xi\right)\left(\int_{a}^{x} \frac{d \xi}{p(\xi, t)}\right)\right\} .
\end{aligned}
$$

Hence, we can write $P^{\prime \prime}(t) \varrho_{\varepsilon}^{-2} u$ as follows:

$$
P^{\prime \prime}(t) \varrho_{\varepsilon}^{-2} u=P^{\prime \prime}(t) P^{-1}(t) P(t) \varrho_{\varepsilon}^{-2} u=\Pi(t) P(t) \varrho_{\varepsilon}^{-2} u=\Pi(t) g,
$$

where

$$
\begin{aligned}
\Pi(t) g= & \left(\frac{\partial^{3} p}{\partial t^{2} \partial x} \frac{1}{p}-\frac{\partial^{2} p}{\partial t^{2}} \frac{1}{p^{2}} \frac{\partial p}{\partial x}\right) \\
& \times\left(\int_{a}^{x} g(\xi, t) d \xi-\frac{\left(\int_{a}^{b}((a-x)(b-x) / p(x, t)) d x \int_{a}^{x} g(\xi, t) d \xi\right)}{\int_{a}^{b}((a-x)(b-x) / p(x, t)) d x}\right)+\frac{\partial^{2} p}{\partial t^{2}} \frac{1}{p} g .
\end{aligned}
$$


Therefore, (4.14) becomes

$$
\begin{aligned}
\int_{Q} u & \frac{\partial^{2}\left(\varrho_{\varepsilon}^{-2}\right)^{*} \mathfrak{I}_{x}^{*}\left(\mathfrak{I}_{\xi} \omega\right)}{\partial t^{2}} d x d t \\
& =\int_{Q}\left(P(t) \varrho_{\varepsilon}^{-2} u+\varepsilon \Pi(t) P(t) \varrho_{\varepsilon}^{-2} u\right)\left(\varrho_{\varepsilon}^{-2}\right)^{*} \mathfrak{J}_{x}^{*}\left(\mathfrak{J}_{\xi} \omega\right) d x d t \\
& =\int_{Q} P(t) \varrho_{\varepsilon}^{-2} u \cdot \Lambda_{\varepsilon}\left(\varrho_{\varepsilon}^{-2}\right)^{*} \mathfrak{J}_{x}^{*}\left(\mathfrak{J}_{\xi} \omega\right) d x d t,
\end{aligned}
$$

where

$$
\begin{aligned}
& \Lambda_{\varepsilon}\left(\varrho_{\varepsilon}^{-2}\right)^{*}=\left(I+\varepsilon \Pi^{*}\right)\left(\varrho_{\varepsilon}^{-2}\right)^{*} \\
\Pi^{*}\left(\varrho_{\varepsilon}^{-2}\right)^{*} \mathfrak{J}_{x}^{*}\left(\mathfrak{J}_{\xi} \omega\right)= & \int_{x}^{b}\left(\frac{\partial^{3} p}{\partial \xi \partial t^{2}} \frac{1}{p}-\frac{\partial^{2} p}{\partial t^{2}} \frac{\partial p}{\partial \xi} \frac{1}{p^{2}}\right)\left(\varrho_{\varepsilon}^{-2}\right)^{*} \mathfrak{J}_{\xi}^{*}\left(\mathfrak{J}_{\zeta} \omega\right) d \xi \\
& -\frac{\int_{x}^{b}((a-\xi)(b-\xi) / p(\xi, t)) d \xi}{\int_{a}^{b}((a-x)(b-x) / p(x, t)) d x} \\
& \times \int_{a}^{b}\left(\frac{\partial^{3} p}{\partial x \partial t^{2}} \frac{1}{p}-\frac{\partial^{2} p}{\partial t^{2}} \frac{\partial p}{\partial x} \frac{1}{p^{2}}\right)\left(\varrho_{\varepsilon}^{-2}\right)^{*} \mathfrak{I}_{x}^{*}\left(\mathfrak{J}_{\xi} \omega\right) d x \\
& +\frac{\partial^{2} p}{\partial t^{2}} \frac{1}{p}\left(\varrho_{\varepsilon}^{-2}\right)^{*} \mathfrak{I}_{x}^{*}\left(\mathfrak{J}_{\xi} \omega\right) .
\end{aligned}
$$

Since the left-hand side of (4.18) is a continuous linear functional of $u$, then the function $\Lambda_{\varepsilon}$ has $\partial \Lambda_{\varepsilon} / \partial x$ and $\partial^{2} \Lambda_{\varepsilon} / \partial x^{2}$ belonging to $L^{2}(Q)$, and such that

$$
\begin{gathered}
\left.\Lambda_{\varepsilon}\right|_{x=a}=\left.\Lambda_{\varepsilon}\right|_{x=b}=0, \\
\left.\frac{\partial \Lambda_{\varepsilon}}{\partial x}\right|_{x=a}=\left.\frac{\partial \Lambda_{\varepsilon}}{\partial x}\right|_{x=b}=0 .
\end{gathered}
$$

For sufficiently small $\varepsilon$, we have $\left\|\varepsilon \Pi^{*}\right\|_{L^{2}(Q)}<1$, from which we conclude that the operator $\Lambda_{\varepsilon}$ possesses a continuous inverse on $L^{2}(Q)$, that is, $\mathfrak{J}_{x}^{*}\left(\mathfrak{J}_{\xi} \omega\right) \in L^{2}(Q)$. We differentiate $\Lambda_{\varepsilon}$ with respect to $x$ :

$$
\frac{\partial \Lambda_{\varepsilon}}{\partial x}\left(\varrho_{\varepsilon}^{-2}\right)^{*} \mathfrak{J}_{x}^{*}\left(\mathfrak{J}_{\xi} \omega\right)=-\left(I+\varepsilon \Pi^{*}\right)\left(\varrho_{\varepsilon}^{-2}\right)^{*} \mathfrak{I}_{x} \omega+\varepsilon \frac{\partial \Pi^{*}}{\partial x}\left(\varrho_{\varepsilon}^{-2}\right)^{*} \mathfrak{I}_{x}^{*}\left(\mathfrak{J}_{\xi} \omega\right)
$$

where

$$
\begin{aligned}
\frac{\partial \Pi^{*}}{\partial x}\left(\varrho_{\varepsilon}^{-2}\right)^{*} \mathfrak{I}_{x}^{*}\left(\mathfrak{J}_{\xi} \omega\right)= & \frac{(a-x)(b-x) / p(x, t)}{\int_{a}^{b}((a-x)(b-x) / p(x, t))} \\
& \times \int_{a}^{b}\left(\frac{\partial^{3} p}{\partial x \partial t^{2}} \frac{1}{p}-\frac{\partial^{2} p}{\partial t^{2}} \frac{\partial p}{\partial x} \frac{1}{p^{2}}\right)\left(\varrho_{\varepsilon}^{-2}\right)^{*} \mathfrak{J}_{x}^{*}\left(\mathfrak{J}_{\xi} \omega\right)
\end{aligned}
$$

Relation (4.23) implies that $\partial \Pi^{*}(t) / \partial x$ is bounded on $L^{2}(Q)$; thus according to (4.22), we deduce that $\mathfrak{J}_{x} \omega \in L^{2}(Q)$ for sufficiently small $\varepsilon$; in other words, $\omega \in L^{2}\left(0, T ; B_{2}^{1}(a\right.$, $b$ ) ) for sufficiently small $\varepsilon$. Similarly, using the boundedness of $\partial^{2} \Pi^{*}(t) / \partial x^{2}$ on $L^{2}(Q)$, we conclude that $\omega \in L^{2}(Q)$. 
According to (4.19) and (4.20), we have

$$
\begin{gathered}
\left.\left(I+\varepsilon \frac{1}{p} \frac{\partial^{2} p}{\partial t^{2}}\right)\left(\varrho_{\varepsilon}^{-2}\right)^{*} \mathfrak{J}_{x}^{*}\left(\mathfrak{J}_{\xi} \omega\right)\right|_{x=a}=0, \\
\left.\left(I+\varepsilon \frac{1}{p} \frac{\partial^{2} p}{\partial t^{2}}\right)\left(\varrho_{\varepsilon}^{-2}\right)^{*} \mathfrak{J}_{x}^{*}\left(\mathfrak{J}_{\xi} \omega\right)\right|_{x=b}=0, \\
\left.\left(I+\varepsilon \frac{1}{p} \frac{\partial^{2} p}{\partial t^{2}}\right)\left(\varrho_{\varepsilon}^{-2}\right)^{*} \mathfrak{J}_{x} \omega\right|_{x=a}=0, \\
\left.\left(I+\varepsilon \frac{1}{p} \frac{\partial^{2} p}{\partial t^{2}}\right)\left(\varrho_{\varepsilon}^{-2}\right)^{*} \mathfrak{J}_{x} \omega\right|_{x=b}=0 .
\end{gathered}
$$

Analogously, for fixed $x \in[a, b]$ and sufficiently small $\varepsilon$, the norm of $\left(\varepsilon(1 / p)\left(\partial^{2} p / \partial t^{2}\right)\right)$ on $L^{2}(0, T)$ is smaller than 1 . Therefore, the operator $\left(I+\varepsilon(1 / p)\left(\partial^{2} p / \partial t^{2}\right)\right)$ has a continuous inverse on $L^{2}(0, T)$. Then, from (4.23), (4.24), (4.25), and (4.26), we get

$$
\begin{gathered}
\left.\mathfrak{J}_{x}^{*}\left(\mathfrak{J}_{\xi} \omega\right)\right|_{x=a}=0, \\
\left.\mathfrak{J}_{x} \omega\right|_{x=b}=0 .
\end{gathered}
$$

We will now construct the function $\omega$. For this, we introduce the function

$$
v(x, t)=e^{c t}\left(c \omega+\frac{\partial \omega}{\partial t}\right)
$$

with

$$
\omega(x, 0)=0
$$

where

$$
c \in\left[\frac{c_{2}+\sqrt{c_{2}^{2}+8 c_{3}^{2}}}{c_{0}},+\infty[.\right.
$$

Solving the differential equation (4.29) with respect to $t$, by taking into account (4.30), we obtain

$$
\omega(x, t)=-e^{-c t} \mathfrak{J}_{t} v .
$$

It follows from relations (4.27), (4.28), and (4.32) that

$$
\begin{aligned}
& \mathfrak{J}_{b} v=0, \\
& \mathfrak{I}_{b}^{2} v=0 .
\end{aligned}
$$

Substituting (4.32) into (4.11) yields

$$
-\left(\frac{\partial^{2} u}{\partial t^{2}}, e^{-c t} \mathfrak{J}_{t} v\right)_{L^{2}\left(0, T ; B_{2}^{1}(a, b)\right)}=-\left(P u, e^{-c t} \mathfrak{J}_{t} v\right)_{L^{2}\left(0, T ; B_{2}^{1}(a, b)\right)}
$$

In identity (4.35), we set

$$
u=\mathfrak{J}_{t}^{2} v,
$$


from which we have

$$
-\left(v, e^{-c t} \mathfrak{J}_{t} v\right)_{L^{2}\left(0, T ; B_{2}^{1}(a, b)\right)}=-\left(P \mathfrak{J}_{t}^{2} v, e^{-c t} \mathfrak{J}_{t} v\right)_{L^{2}\left(0, T ; B_{2}^{1}(a, b)\right)} .
$$

Integrating by parts both sides of (4.37), we get

$$
\begin{aligned}
e^{-c T}\left\|\mathfrak{I}_{T} v\right\|_{B_{2}^{1}(a, b)}^{2}+c\|v\|_{B_{2}^{1, w}\left(0, T ; B_{2}^{1}(a, b)\right)}^{2} & \\
= & -\int_{a}^{b} e^{-c T} p(x, T)\left(\mathfrak{I}_{T} v\right)^{2} d x-\int_{Q} e^{-c t}\left(c p-\frac{\partial p}{\partial t}\right)\left(\mathfrak{I}_{t} v\right)^{2} d x d t \\
& -2 \int_{Q} e^{-c t} \frac{\partial p}{\partial x} \mathfrak{I}_{t}^{2} v \mathfrak{I}_{x}\left(\mathfrak{J}_{t} v\right) d x d t .
\end{aligned}
$$

The last integral on the right-hand side of (4.38) is dominated by

$$
\frac{c}{2}\|v\|_{B_{2}^{1, w}\left(0, T ; B_{2}^{1}(a, b)\right)}^{2}+\frac{2}{c} \int_{Q} e^{-c t}\left(\frac{\partial p}{\partial x}\right)^{2}\left(\mathfrak{J}_{t} v\right)^{2} d x d t
$$

where the first term is absorbed in the left-hand side. It then follows, by omitting the first term of each side of (4.38) and by using Assumption 2.1, that

$$
\frac{c^{2}}{2}\|v\|_{B_{2}^{1, w}\left(0, T ; B_{2}^{1}(a, b)\right)}^{2} \leq-\left(c^{2} c_{0}-c c_{2}-2 c_{3}^{2}\right)\|v\|_{B_{2}^{2}\left(0, T ; L^{2}(a, b)\right)}^{2} .
$$

According to (4.31), we deduce that $v$ and thus $\omega$ vanish almost everywhere in $Q$. This achieves the proof of Proposition 4.2.

This completes the proof of Theorem 4.1.

ACKNOWLEDGMENT. This work has been done in the Abdus Salam International Centre of Theoretical Physics, Trieste, Italy. This work was supported by Laboratoire de matériaux et structure des systémes électromécaniques et leurs fiabilités, Centre Universitaire Larbi Ben M’Hidi, Oum El Bouagui, Algeria.

\section{REFERENCES}

[1] R. A. Adams, Sobolev Spaces, Pure and Applied Mathematics, vol. 65, Academic Press, New York, 1975.

[2] S. A. Beilin, Existence of solutions for one-dimensional wave equations with nonlocal conditions, Electron. J. Differential Equations 2001 (2001), no. 76, 1-8.

[3] A. Bouziani, Problèmes mixtes avec conditions intégrales pour quelques équations aux dérivées partielles, Ph.D. thesis, Constantine University, 1996.

[4] S_ Solution forte d'un problème mixte avec une condition non locale pour une classe d'équations hyperboliques [Strong solution of a mixed problem with a nonlocal condition for a class of hyperbolic equations], Acad. Roy. Belg. Bull. Cl. Sci. (6) 8 (1997), no. 1-6, 53-70 (French).

[5] _ Initial-boundary value problem with a nonlocal condition for a viscosity equation, Int. J. Math. Math. Sci. 30 (2002), no. 6, 327-338.

[6] _ On the solvability of parabolic and hyperbolic problems with a boundary integral condition, Int. J. Math. Math. Sci. 31 (2002), no. 4, 201-213.

[7] D. G. Gordeziani and G. A. Avalishvili, Solution of nonlocal problems for one-dimensional oscillations of a medium, Mat. Model. 12 (2000), no. 1, 94-103 (Russian). 
[8] N. I. Jurčuk, A partially characteristic boundary value problem for a certain form of partial differential equations. II, Differ. Uravn. 5 (1969), 531-542 (Russian).

[9] __ Solvability of boundary value problems for certain operator-differential equations, Differ. Uravn. 13 (1977), no. 4, 626-636 (Russian).

[10] _ A mixed problem with an integral condition for some parabolic equations, Differ. Uravn. 22 (1986), no. 12, 2117-2126 (Russian).

[11] O. A. Ladyženskaya, On the solution of non-stationary operator equations, Mat. Sb. (N.S.) 39(81) (1956), 491-524.

[12] A. M. Nakhushev, An approximate method for solving boundary value problems for differential equations and its application to the dynamics of ground moisture and ground water, Differ. Uravn. 18 (1982), no. 1, 72-81 (Russian).

[13] L. S. Pulkina, A non-local problem with integral conditions for hyperbolic equations, Electron. J. Differential Equations 1999 (1999), no. 45, 1-6.

[14]__ On the solvability in $L_{2}$ of a nonlocal problem with integral conditions for a hyperbolic equation, Differ. Equ. 36 (2000), no. 2, 316-318.

Abdelfatah Bouziani: Département de Mathématiques, Centre Universitaire Larbi Ben M’hidi, Oum El Bouagui 04000, Algeria 


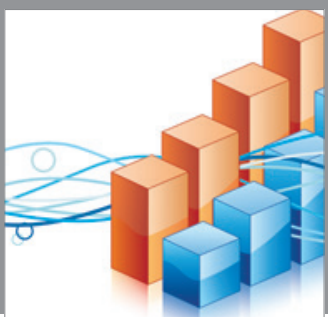

Advances in

Operations Research

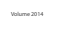

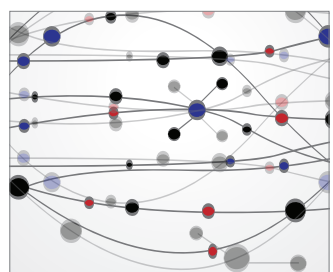

\section{The Scientific} World Journal
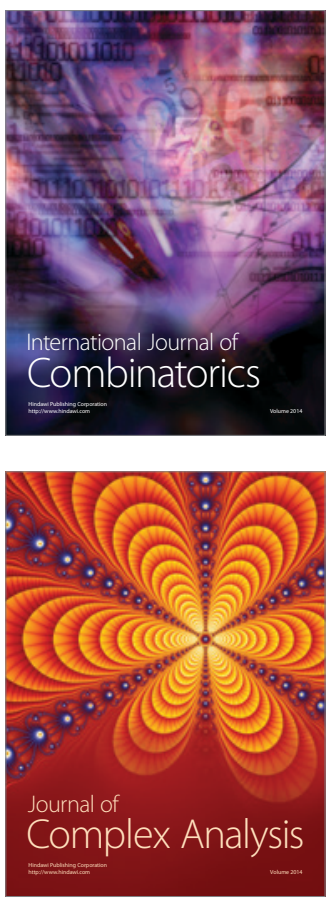

International Journal of

Mathematics and

Mathematical

Sciences
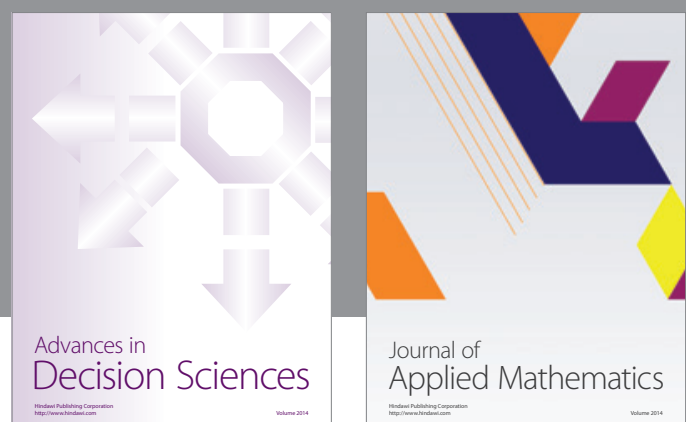

Journal of

Applied Mathematics
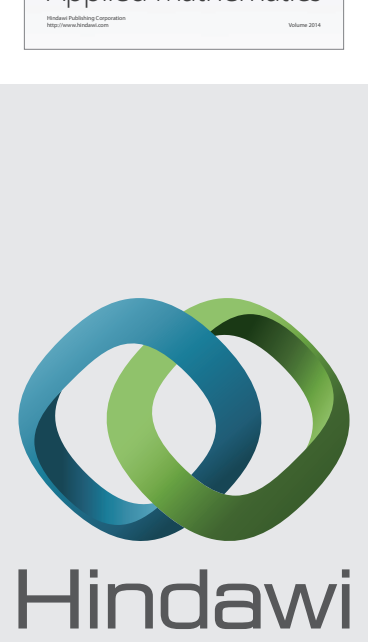

Submit your manuscripts at http://www.hindawi.com
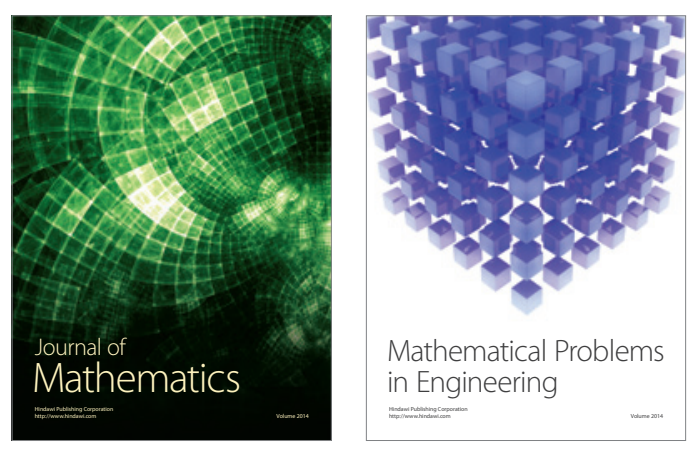

Mathematical Problems in Engineering
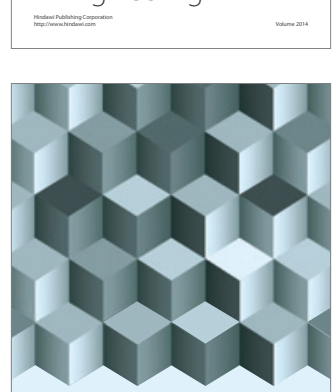

Journal of

Function Spaces
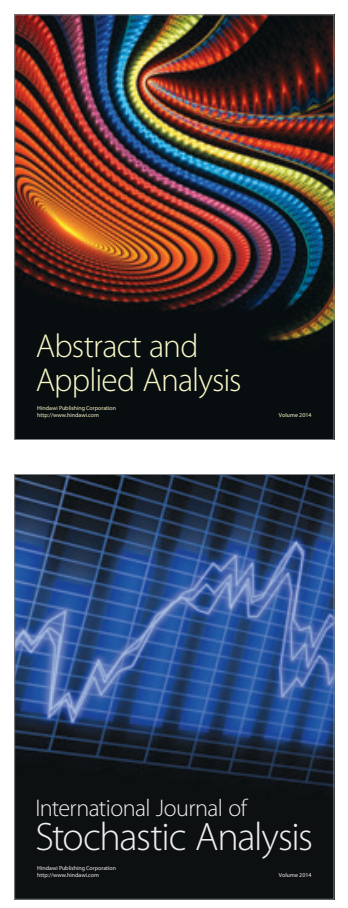

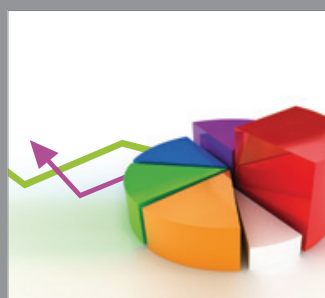

ournal of

Probability and Statistics

Promensencen
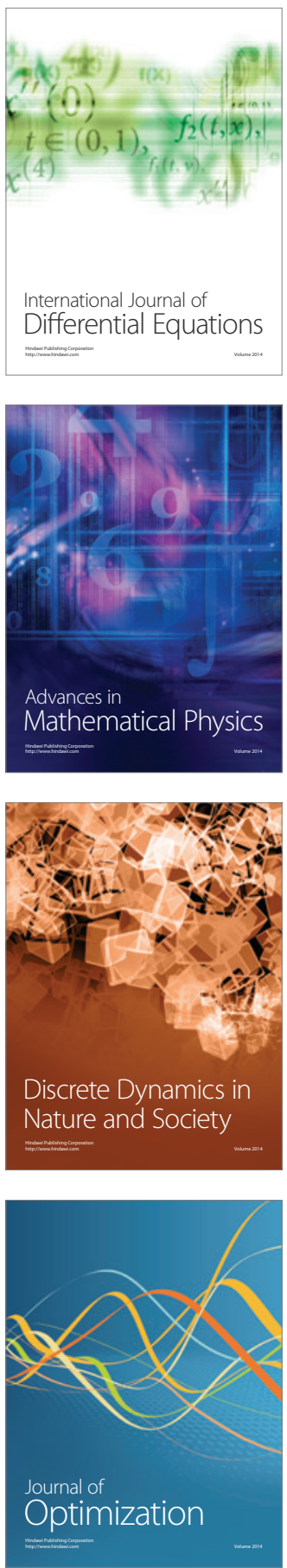